\title{
On the Well-posedness of Some Generalized Characteristic Cauchy Problems
}

\author{
J..-A. Marti ${ }^{*}$, V. Dévoué ${ }^{1}$, A. Delcroix ${ }^{2}$ \\ E. Allaud ${ }^{1}$, H. Vernaeve ${ }^{3}$ \\ ${ }^{1}$ Laboratoire CEREGMIA, Université des Antilles, Campus de Shoelcher \\ BP 7209, 97275 Schoelcher Cedex, Martinique \\ ${ }^{2}$ Laboratoire CRREF, IUFM de Guadeloupe, Morne Ferret, BP 399, 97178 Abymes Cedex, Guadeloupe \\ ${ }^{3}$ Department of Mathematics, Ghent University, Building S22, Krijgslaan 281, B 9000 Gent, Belgium
}

\begin{abstract}
By means of convenient regularization for an ill posed Cauchy problem, we define an associated generalized problem and discuss the conditions for the solvability of it. To illustrate this, starting from the semilinear unidirectional wave equation with data given on a characteristic curve, we show existence and uniqueness of the solution.
\end{abstract}

Keywords and phrases: regularization of data, regularization of data, regularisation of problems, distributions, nonlinear generalized functions,, nonlinear problems, characteristic problems, transport equation

Mathematics Subject Classification: 35A01, 35A02, 35A25, 35D99, 46F30, 46T30

\section{Introduction}

Many obstructions can be encountered when trying to solve a Cauchy problem for PDEs with the data given on a characteristic manifold, and, a fortiori, to obtain uniqueness or well-posedness in Hadamard sense. We can refer to many works inspired in the complex field by the ideas of Gårding, Kotake, Leray [10] and others on the continuation of holomorphic solutions and, in the real field, by the ideas of Egorov [9], Hörmander [13] and others on the distribution solutions of some Cauchy problems supported in a half space whose boundary is a characteristic hyperplane.

Here, we propose another method, based on a parametrized family of geometric transformations of the characteristic manifold, in continuation of previous ideas developed in $[5-8,12,15]$. In order to concentrate on the methods and not on the technicalities, we consider the Cauchy problem for a simple equation, namely the transport equation (in basic form)

$$
\frac{\partial u}{\partial t}=F(., ., u) ;\left.\quad u\right|_{\gamma}=v
$$

where $\gamma$ of equation $x=0$ is obviously globally characteristic for the Cauchy problem.

${ }^{*}$ Corresponding author. E-mail: jamarti@univ-ag.fr 
For focusing only on the characteristic singularity, $v$ and $F$ are supposed to be smooth. Moreover $F$ has to verify some estimates involving the derivatives. Clearly $\left(P_{c}\right)$ is ill-posed but can be associated to a generalized problem

$$
P(D) u=\mathcal{F}(u) ; \quad \mathcal{R}(u)=v
$$

well formulated in convenient algebras of nonlinear generalized functions, by means of generalized operators: $\mathcal{F}$, associated to $F$, and $\mathcal{R}$, obtained by replacing the characteristic curve $\gamma$ by a family $\left(\gamma_{\varepsilon}\right)_{\varepsilon}$ of non-characteristic ones of equation $x=l_{\varepsilon}(t)$ where $\left(l_{\varepsilon}\right)_{\varepsilon}$ is a regularizing family. We can show the existence of a generalized solution in some $(\mathcal{C}, \mathcal{E}, \mathcal{P})$-algebra [14] $\mathcal{A}\left(\mathbb{R}^{2}\right)$ based on the space of smooth functions. Independence of this solution with respect to some "tempered" class represented by $\left(l_{\varepsilon}\right)_{\varepsilon}$ can also be established under some additional assumption on the growth of $\left(l_{\varepsilon}\right)_{\varepsilon}$. However this generalized solution in $\mathcal{A}\left(\mathbb{R}^{2}\right)$ fails to be, in general, unique. We show how uniqueness may be recovered by searching a solution in the space of new tempered generalized functions $\mathcal{G}_{\mathcal{O}_{M}}\left(\mathbb{R}^{2}\right)$ based on the space of slowly increasing smooth functions [4] in which pointwise characterization exists [20].

\section{General overview on $(\mathcal{C}, \mathcal{E}, \mathcal{P})$-type algebras}

\subsection{Algebraic and topological structures}

We begin by recalling the notions from $[14,15]$ that form the basis for our study. Let:

(1) $\Lambda$ be a set of indices;

(2) $A$ a solid subring of the $\operatorname{ring} \mathbb{K}^{\Lambda},(\mathbb{K}=\mathbb{R}$ or $\mathbb{C})$, that is $A$ has the following stability property: whenever $\left(\left|s_{\lambda}\right|\right)_{\lambda} \leq\left(r_{\lambda}\right)_{\lambda}$ (i.e. for any $\left.\lambda,\left|s_{\lambda}\right| \leq r_{\lambda}\right)$ for any pair $\left(\left(s_{\lambda}\right)_{\lambda},\left(r_{\lambda}\right)_{\lambda}\right) \in \mathbb{K}^{\Lambda} \times|A|$, it follows that $\left(s_{\lambda}\right)_{\lambda} \in A$, with $|A|=\left\{\left(\left|r_{\lambda}\right|\right)_{\lambda}:\left(r_{\lambda}\right)_{\lambda} \in A\right\}$ and $I_{A}$ a solid ideal of $A$;

(3) $\mathcal{E}$ be a sheaf of $\mathbb{K}$-topological algebras over a topological space $X$.

Moreover, suppose that:

(4) For any open set $\Omega$ in $X$, the algebra $\mathcal{E}(\Omega)$ is endowed with a family $\mathcal{P}(\Omega)=\left(P_{i}\right)_{i \in I(\Omega)}$ of semi-norms such that if $\Omega_{1} \subset \Omega_{2}$ are two open subsets of $X$, it follows that $I\left(\Omega_{1}\right) \subset I\left(\Omega_{2}\right)$ and if $\rho_{1}^{2}$ is the restriction operator $\mathcal{E}\left(\Omega_{2}\right) \rightarrow \mathcal{E}\left(\Omega_{1}\right)$, then, for each $P_{i} \in \mathcal{P}\left(\Omega_{1}\right)$ the semi-norm $\widetilde{P}_{i}=P_{i} \circ \rho_{1}^{2}$ extends $P_{i}$ to $\mathcal{P}\left(\Omega_{2}\right)$;

(5) Let $\Theta=\left(\Omega_{h}\right)_{h \in H}$ be any family of open sets in $X$ with $\Omega=\cup_{h \in H} \Omega_{h}$. Then, for each $P \in \mathcal{P}(\Omega)$, there exists a finite subfamily $\left(\Omega_{j}\right)_{1 \leq j \leq n(i)}$ of $\Theta$ and corresponding semi-norms $P_{j} \in \mathcal{P}\left(\Omega_{j}\right)(1 \leq j \leq n(i))$ such that, for any $u \in \mathcal{E}(\Omega), P(u) \leq \sum_{j=1}^{n(i)} P_{j}\left(\left.u\right|_{\Omega_{j}}\right)$.

Define $|B|=\left\{\left(\left|r_{\lambda}\right|\right)_{\lambda} \mid\left(r_{\lambda}\right)_{\lambda} \in B\right\}$ for $B=A, I_{A}$. Set $\mathcal{C}=A / I$ and let $\mathcal{H}_{(A, \mathcal{E}, \mathcal{P})}(\Omega)\left(\operatorname{resp} . \mathcal{J}_{\left(I_{A}, \mathcal{E}, \mathcal{P}\right)}(\Omega)\right)$ be the set of all $\left(u_{\lambda}\right)_{\lambda} \in[\mathcal{E}(\Omega)]^{\Lambda}$ such that $\left(\left(P_{i}\left(u_{\lambda}\right)\right)_{\lambda} \in|A|\right.$ (resp. $\left.\left|I_{A}\right|\right)$ for all $i \in I(\Omega)$.

Note that, from (2), $|A|$ is a subset of $A$ and that $A_{+}=\left\{\left(b_{\lambda}\right)_{\lambda} \in A \mid(\forall \lambda \in \Lambda)\left(b_{\lambda} \geq 0\right)\right\}=|A|$. The same holds for $I_{A}$. Furthermore, $(2)$ implies also that $A$ is a $\mathbb{K}$-algebra. From $[14,15]$, we get that $\mathcal{H}_{(A, \mathcal{E}, \mathcal{P})}$ (resp. $\mathcal{J}_{\left(I_{A}, \mathcal{E}, \mathcal{P}\right)}$ ) is a sheaf of $\mathbb{K}$-subalgebras (resp. of ideals) of the sheaf $\mathcal{E}^{\Lambda}$ (resp. of $\mathcal{H}_{(A, \mathcal{E}, \mathcal{P})}$ ) and that the factor $\mathcal{H}_{(A, \mathcal{E}, \mathcal{P})} / \mathcal{J}_{\left(I_{A}, \mathcal{E}, \mathcal{P}\right)}$ is a presheaf with localization principle in addition. Moreover, the constant sheaf $\mathcal{H}_{(A, \mathbb{K},|.|)} / \mathcal{J}_{\left(I_{A}, \mathbb{K},|.|\right)}$ is equal to the sheaf $\mathcal{C}=A / I_{A}$.

We call presheaf of $(\mathcal{C}, \mathcal{E}, \mathcal{P})$-algebra, the factor presheaf of the algebras $\mathcal{A}=\mathcal{H}_{(A, \mathcal{E}, \mathcal{P})} / \mathcal{J}_{\left(I_{A}, \mathcal{E}, \mathcal{P}\right)}$ over the ring $\mathcal{C}=A / I_{A}$ and we denote by $\left[u_{\lambda}\right]$ the class in $\mathcal{A}(\Omega)$ of $\left(u_{\lambda}\right)_{\lambda \in \Lambda} \in \mathcal{H}_{(A, \mathcal{E}, \mathcal{P})}(\Omega)$.

Note 2.1. For any topological space $T, K \Subset T$ means that $K$ is a compact subset of $T$.

Example 2.2. (Special Colombeau Algebra $[2,11,17]$ ) We consider the sheaf $\mathcal{E}=\mathrm{C}^{\infty}$ over $\mathbb{R}^{d}$, where $\mathcal{P}$ is the usual family of topologies $\left(\mathcal{P}_{\Omega}\right)_{\Omega \in \mathcal{O}\left(\mathbb{R}^{d}\right)}$. Here $\mathcal{O}\left(\mathbb{R}^{d}\right)$ denotes the set of all open sets of $\mathbb{R}^{d}$. Let us recall that $\mathcal{P}_{\Omega}$ is defined by the family of semi-norms $\left(p_{K, l}\right)_{K \Subset \Omega, l \in \mathbb{N}}$ with

$$
\forall f \in \mathrm{C}^{\infty}(\Omega), \quad p_{K, l}(f)=\sup _{x \in K,|\alpha| \leq l}\left|D^{\alpha} f(x)\right| .
$$


Let $A$ (resp. $I$ ) be the set of all $\left(r_{\varepsilon}\right)_{\varepsilon} \in \mathbb{R}^{(0,1]}$ such that there exists $m \in \mathbb{N}$ (resp. for all $q \in \mathbb{N}$ ) with $\left|r_{\varepsilon}\right|=\mathrm{o}\left(\varepsilon^{-m}\right)$ (resp. $\left.\quad\left|r_{\varepsilon}\right|=\mathrm{o}\left(\varepsilon^{q}\right)\right)$ as $\varepsilon \rightarrow 0$. The sheaf $\mathcal{A}=\mathcal{H}_{(A, \mathcal{E}, \mathcal{P})} / \mathcal{J}_{\left(I_{A}, \mathcal{E}, \mathcal{P}\right)}$ is the sheaf of (special) Colombeau algebras $\mathcal{G}$. In this case, we shall write $\mathcal{H}_{(A, \mathcal{E}, \mathcal{P})}=\mathcal{X}$ and $\mathcal{J}_{\left(I_{A}, \mathcal{E}, \mathcal{P}\right)}=\mathcal{N}$.

We refer to $[5,14]$ for a complete discussion about embedding of $(\mathcal{C}, \mathcal{E}, \mathcal{P})$-algebras into spaces of smooth functions and distributions. From now on we assume that $A$ is a ring with unity and $\Lambda$ is left-filtering for a given (partial) order relation $\prec$.

Remark 2.3. (An association process) Consider $\Omega$ an open subset of $X, \mathcal{F}$ a given sheaf of topological $\mathbb{K}$-vector spaces (resp. $\mathbb{K}$-algebras) over $X$ containing $\mathcal{E}$ as a subsheaf and $a: \mathbb{R}_{+} \rightarrow A_{+}$a map such that $a(0)=1$ (for $r \in \mathbb{R}_{+}$, we denote $a(r)$ by $\left.\left(a_{\lambda}(r)\right)_{\lambda}\right)$.

For $\left(v_{\lambda}\right)_{\lambda} \in \mathcal{H}_{(A, \mathcal{E}, \mathcal{P})}(\Omega)$, we shall denote by $\lim _{\Lambda, \mathcal{F}(\Omega)} v_{\lambda}$ the limit of $\left(v_{\lambda}\right)_{\lambda}$ for the $\mathcal{F}$-topology when it exists. We recall that $\left.\lim _{\Lambda, \mathcal{F}(\Omega)} u_{\lambda}\right|_{V}=f \in \mathcal{F}(V)$ iff, for each $\mathcal{F}$-neighborhood $W$ of $f$, there exists $\lambda_{0} \in \Lambda$ such that: $\lambda \prec \lambda_{0} \Longrightarrow u_{\lambda} \in W$. We also assume that, for each open subset $V \subset \Omega$, we have

$$
\mathcal{J}_{\left(I_{A}, \mathcal{E}, \mathcal{P}\right)}(V) \subset\left\{\left(v_{\lambda}\right)_{\lambda} \in \mathcal{H}_{(A, \mathcal{E}, \mathcal{P})}(V) \mid \lim _{\Lambda, \mathcal{F}(\Omega)} v_{\lambda}=0\right\} .
$$

Consider $u=\left[u_{\lambda}\right] \in \mathcal{A}(\Omega), r \in \mathbb{R}_{+}, V$ an open subset of $\Omega$ and $f \in \mathcal{F}(V)$. We say that $u$ is $a(r)$ associated to $f$ in $V$, denoted by $u \underset{\mathcal{F}(V)}{\stackrel{a(r)}{\sim}} f$, if $\lim _{\Lambda, \mathcal{F}(\Omega)}\left(\left.a_{\lambda}(r) u_{\lambda}\right|_{V}\right)=f$. In particular, if $r=0, u$ and $f$ are said to be associated in $V$.

Example 2.4. Take $\left.\left.X=\mathbb{R}^{d}, \mathcal{F}=\mathcal{D}^{\prime}, \Lambda=\right] 0,1\right], \mathcal{A}=\mathcal{G}, V=\Omega, r=0$. The usual association [11, §1.2.6] between $u=\left[u_{\varepsilon}\right] \in \mathcal{G}(\Omega)$ and $T \in \mathcal{D}^{\prime}(\Omega)$ is defined by

$$
u \sim T \Longleftrightarrow u \underset{\mathcal{D}^{\prime}(\Omega)}{\stackrel{a(0)}{\sim}} T \Longleftrightarrow \lim _{\varepsilon \rightarrow 0, \mathcal{D}^{\prime}(\Omega)} u_{\varepsilon}=T .
$$

In practice, the ring $A$ and the ideal $I_{A}$ are constructed as follows. Let $B_{p}$ a finite family of $p$ nets in $\left(\mathbb{R}_{+}^{*}\right)^{\Lambda}$ (usually given by the asymptotic structure of the problem). Consider $B$ the subset of elements in $\left(\mathbb{R}_{+}^{*}\right)^{\Lambda}$ obtained as rational fractions with coefficients in $\mathbb{R}_{+}^{*}$, of elements in $B_{p}$ as variables. Define

$$
A=\left\{\left(a_{\lambda}\right)_{\lambda} \in \mathbb{K}^{\Lambda} \mid\left(\exists\left(b_{\lambda}\right)_{\lambda} \in B\right)\left(\exists \lambda_{0} \in \Lambda\right)\left(\forall \lambda \prec \lambda_{0}\right)\left(\left|a_{\lambda}\right| \leq b_{\lambda}\right)\right\} .
$$

We say that $A$ is overgenerated by $B_{p}$ (and it is easy to see that $A$ is a solid subring of $\mathbb{K}^{\Lambda}$ ). If $I_{A}$ is some solid ideal of $A$, we also say that $\mathcal{C}=A / I_{A}$ is overgenerated by $B_{p}$. As a "canonical" ideal of $A$, we usually choose

$$
I_{A}=\left\{\left(a_{\lambda}\right)_{\lambda} \in \mathbb{K}^{\Lambda} \mid\left(\forall\left(b_{\lambda}\right)_{\lambda} \in B\right)\left(\exists \lambda_{0} \in \Lambda\right)\left(\forall \lambda \prec \lambda_{0}\right)\left(\left|a_{\lambda}\right| \leq b_{\lambda}\right)\right\} .
$$

In this paper, we shall consider the particular case $\mathcal{E}=\mathrm{C}^{\infty}$ with $X=\mathbb{R}^{d}$ and the usual topology given by the family of semi norms $\left(P_{K, l}\right)_{K \Subset \Omega, l \in \mathbb{N}}$ defined by (2.1). We shall construct later the asymptotic structure given by $\mathcal{C}=A / I_{A}$, in relationship with the regularization of the ill posed problem. However, for any choice of $\mathcal{C}$, we recall that $\mathcal{A}$ is a sheaf of differential algebras with $D^{\alpha} u=\left[D^{\alpha} u_{\lambda}\right]$ where $\left(u_{\lambda}\right)_{\lambda} \in u$. For $\left(\mathcal{C}, C^{\infty}, \mathcal{P}\right)$-algebras, we have the analogue of [11, Thm 1.2.3]:

Proposition 2.5. [3] Assume that the set B, defined above, is stable by inverse and that there exists $\left(a_{\lambda}\right)_{\lambda} \in B$ with $\lim _{\Lambda} a_{\lambda}=0$. Consider $\left(u_{\lambda}\right)_{\lambda} \in \mathcal{H}_{(A, \mathcal{E}, \mathcal{P})}\left(\mathbb{R}^{d}\right)$ such that, for all $K \Subset \mathbb{R}^{d},\left(P_{K, 0}\left(u_{\lambda}\right)\right)_{\lambda} \in$ $\left|I_{A}\right|$. Then $\left(u_{\lambda}\right)_{\lambda} \in \mathcal{J}_{(A, \mathcal{E}, \mathcal{P})}\left(\mathbb{R}^{d}\right)$.

In the sequel, we shall also consider the algebra of tempered generalized functions. For $f \in \mathrm{C}^{\infty}\left(\mathbb{R}^{n}\right)$, $r \in \mathbb{Z}$ and $m \in \mathbb{N}$, we set $\mu_{r, m}(f)=\sup _{x \in \mathbb{R}^{n},|\alpha| \leq m}(1+|x|)^{r}\left|\mathcal{D}^{\alpha} f(x)\right|$. Define

$$
\begin{aligned}
\mathcal{M}_{\tau}\left(\mathbb{R}^{n}\right) & =\left\{\left(f_{\varepsilon}\right)_{\varepsilon} \in \mathcal{O}_{M}\left(\mathbb{R}^{n}\right)^{(0,1]} \mid(\forall m \in \mathbb{N})(\exists q \in \mathbb{N})(\exists N \in \mathbb{N}) \quad\left(\mu_{-q, m}\left(f_{\varepsilon}\right)=O\left(\varepsilon^{-N}\right) \text { as } \varepsilon \rightarrow 0\right)\right\}, \\
\mathcal{N}_{\tau}\left(\mathbb{R}^{n}\right) & =\left\{\left(f_{\varepsilon}\right)_{\varepsilon} \in \mathcal{O}_{M}\left(\mathbb{R}^{n}\right)^{(0,1]} \mid(\forall m \in \mathbb{N})(\exists q \in \mathbb{N})(\forall p \in \mathbb{N})\left(\mu_{-q, m}\left(f_{\varepsilon}\right)=O\left(\varepsilon^{p}\right) \text { as } \varepsilon \rightarrow 0\right)\right\} .
\end{aligned}
$$


It is easy to show that $\mathcal{M}_{\tau}\left(\mathbb{R}^{n}\right)$ (resp. $\mathcal{N}_{\tau}\left(\mathbb{R}^{n}\right)$ ) is a subalgebra (resp. ideal) of $\mathcal{O}_{M}\left(\mathbb{R}^{n}\right)^{(0,1]}$ $\left(\operatorname{resp} . \mathcal{M}_{\tau}\left(\mathbb{R}^{n}\right)\right)$. The algebra $\mathcal{G}_{\tau}\left(\mathbb{R}^{n}\right)=\mathcal{M}_{\tau}\left(\mathbb{R}^{n}\right) / \mathcal{N}_{\tau}\left(\mathbb{R}^{n}\right)$ is called the algebra of tempered generalized functions $[11,16]$. The generalized derivation, defined as above for $\left(\mathcal{C}, C^{\infty}, \mathcal{P}\right)$-algebras, provides $\mathcal{G}_{\tau}\left(\mathbb{R}^{n}\right)$ with a differential algebraic structure.

Remark 2.6. (Simplification of notations) In the sequel, we shall have $d=1$ or $d=2$ and take $\Lambda=(0,1]$. We simplify the notations by writing $\mathcal{H}($ resp. $\mathcal{J})$ instead of $\mathcal{H}_{(A, \mathcal{E}, \mathcal{P})}\left(\right.$ resp. $\left.\mathcal{J}_{(A, \mathcal{E}, \mathcal{P})}\right)$. We keep the same sheaf symbols $\mathcal{H}, \mathcal{J}, \mathcal{A}=\mathcal{H} / \mathcal{J}$ for $X=\mathbb{R}^{d}$ or $X=\Omega$, where $d=1,2$ and $\Omega$ is an open subset of $\mathbb{R}^{d}$.

\subsection{Generalized operators and general restrictions}

Let $\Omega$ be an open subset of $\mathbb{R}^{2}$ and $F \in \mathrm{C}^{\infty}(\Omega \times \mathbb{R}, \mathbb{R})$. We say that the algebra $\mathcal{A}(\Omega)$ is stable under $F$ if, for all $\left(u_{\varepsilon}\right)_{\varepsilon} \in \mathcal{H}(\Omega)$ and all $\left(i_{\varepsilon}\right)_{\varepsilon} \in \mathcal{J}(\Omega)$, we have $\left(F\left(\cdot, \cdot, u_{\varepsilon}\right)\right)_{\varepsilon} \in \mathcal{H}(\Omega)$ and $\left(F\left(\cdot, \cdot, u_{\varepsilon}+i_{\varepsilon}\right)-F\left(\cdot, \cdot, u_{\varepsilon}\right)\right)_{\varepsilon} \in \mathcal{J}(\Omega)$. If $\mathcal{A}\left(\mathbb{R}^{2}\right)$ if stable under $F$, for $u=\left[u_{\varepsilon}\right] \in \mathcal{A}\left(\mathbb{R}^{2}\right),\left[F\left(., ., u_{\varepsilon}\right)\right]$ is a well defined element of $\mathcal{A}\left(\mathbb{R}^{2}\right)$ (i.e. not depending on $\left(u_{\varepsilon}\right)_{\varepsilon} \in u$ ).

A simple example of stability condition is when $F$ is smoothly tempered, which means that the following two conditions are satisfied:

(i) For each $K \Subset \mathbb{R}^{2}, l \in \mathbb{N}$ and $u \in \mathrm{C}^{\infty}(\Omega, \mathbb{R})$, there is a positive finite sequence $\left(C_{j}\right)_{1 \leq j \leq l}$ such that $P_{K, l}(F(\cdot, \cdot, u)) \leq \sum_{i=0}^{l} C_{i}\left(P_{K, l}(u)\right)^{i}$,

(ii) For each $K \Subset \mathbb{R}^{2}, l \in \mathbb{N}, u, v \in \mathrm{C}^{\infty}(\Omega, \mathbb{R})$, there is a positive finite sequence $\left(D_{j}\right)_{1 \leq j \leq l}$ such that $P_{K, l}\left(F(\cdot, \cdot, v)-F(\cdot, \cdot, u) \leq \sum_{j=1}^{l} D_{j}\left(P_{K, l}(v-u)\right)^{j}\right.$.

Definition 2.7. [5] If $\mathcal{A}\left(\mathbb{R}^{2}\right)$ if stable under $F$, the map

$$
\mathcal{F}: \mathcal{A}\left(\mathbb{R}^{2}\right) \rightarrow \mathcal{A}\left(\mathbb{R}^{2}\right), \quad u=\left[u_{\varepsilon}\right] \mapsto\left[F\left(., ., u_{\varepsilon}\right)\right]
$$

is called the generalized map corresponding to $F$.

Consider $\left(l_{\varepsilon}\right)_{\varepsilon} \in \mathrm{C}^{\infty}(\mathbb{R})^{\Lambda}$. Set

$$
R_{\varepsilon}: \mathrm{C}^{\infty}\left(\mathbb{R}^{2}\right) \rightarrow \mathrm{C}^{\infty}(\mathbb{R}), g \mapsto R_{\varepsilon}(g)
$$

with

$$
R_{\varepsilon}(g): \mathbb{R} \rightarrow \mathbb{R}, t \mapsto g\left(t, l_{\varepsilon}(t)\right) .
$$

The family $\left(R_{\varepsilon}\right)_{\varepsilon}$ maps $\mathrm{C}^{\infty}\left(\mathbb{R}^{2}\right)^{\Lambda}$ into $\mathrm{C}^{\infty}(\mathbb{R})^{\Lambda}$. We say that the family $\left(l_{\varepsilon}\right)_{\varepsilon}$ is compatible with the generalized restriction if, for all $\left(u_{\varepsilon}\right)_{\varepsilon} \in \mathcal{H}\left(\mathbb{R}^{2}\right)$ (resp. $\left.\left(i_{\varepsilon}\right)_{\varepsilon} \in \mathcal{J}\left(\mathbb{R}^{2}\right)\right),\left(u_{\varepsilon}\left(\cdot, l_{\varepsilon}(\cdot)\right)\right)_{\varepsilon} \in \mathcal{H}(\mathbb{R})$ (resp. $\left.\left(i_{\varepsilon}\left(\cdot, l_{\varepsilon}(\cdot)\right)\right)_{\varepsilon} \in \mathcal{J}(\mathbb{R})\right)$.

Definition 2.8. If the family of smooth functions $\left(l_{\varepsilon}\right)_{\varepsilon}$ is compatible with the generalized restriction, the map

$$
\mathcal{R}: \mathcal{A}\left(\mathbb{R}^{2}\right) \rightarrow \mathcal{A}(\mathbb{R}), \quad u=\left[u_{\varepsilon}\right] \mapsto\left[u_{\varepsilon}\left(\cdot, l_{\varepsilon}(\cdot)\right)\right]=\left[R_{\varepsilon}\left(u_{\varepsilon}\right)\right]
$$

is called the generalized restriction mapping corresponding to the family $\left(l_{\varepsilon}\right)_{\varepsilon}$.

Definition 2.9. [11] Let $\left(f_{\varepsilon}\right)_{\varepsilon} \in \mathrm{C}^{\infty}\left(\mathbb{R}^{n}\right)^{\Lambda}$. We say $\left(l_{\varepsilon}\right)_{\varepsilon}$ is $c$-bounded if for all $K \Subset \mathbb{R}^{n}$, there exists $L \Subset \mathbb{R}^{n}$ such that $l_{\varepsilon}(K) \subset L$ for all $\varepsilon$ ( $L$ is independent of $\varepsilon$ ).

The following proposition establishes a link between the c-boundeness and the compatibility with restriction.

Proposition 2.10. Assume that $\left(l_{\varepsilon}\right)_{\varepsilon}$ belongs to $\mathcal{H}(\mathbb{R})$ and $\left(l_{\varepsilon}\right)_{\varepsilon}$ is c-bounded, then the family $\left(l_{\varepsilon}\right)_{\varepsilon}$ is compatible with second side restriction. 
Proof. As $\left(l_{\varepsilon}\right)_{\varepsilon}$ is $c$-bounded, for each $K \Subset \mathbb{R}$, it exists $K^{\prime} \Subset \mathbb{R}$ such that, for all $\varepsilon \in \Lambda, l_{\varepsilon}(K) \subset K^{\prime}$. Take $\left(u_{\varepsilon}\right)_{\varepsilon} \in \mathcal{H}\left(\mathbb{R}^{2}\right)$ (resp. $\left.\left(i_{\varepsilon}\right)_{\varepsilon} \in \mathcal{J}\left(\mathbb{R}^{2}\right)\right)$ and set $v_{\varepsilon}(t)=u_{\varepsilon}\left(t, l_{\varepsilon}(t)\right)$. Then we have: $p_{K, 0}\left(v_{\varepsilon}\right) \leq P_{K \times K^{\prime}, 0}\left(u_{\varepsilon}\right)$, $p_{K, 1}\left(v_{\varepsilon}\right) \leq P_{K \times K^{\prime},(1,0)}\left(u_{\varepsilon}\right)+P_{K \times K^{\prime},(0,1)}\left(u_{\varepsilon}\right) p_{K, 1}\left(l_{\varepsilon}\right)$. By induction we can see that for each $K \Subset \mathbb{R}$ and each $\rho \in \mathbb{N}, p_{K, \rho}\left(v_{\varepsilon}\right)$ is estimate by sums or products of terms like $P_{K \times K^{\prime},(n, m)}\left(u_{\varepsilon}\right)$ for $n+m \leq \rho$, or $p_{K, k}\left(l_{\varepsilon}\right)$ for $k \leq \rho$. Then, as $\left(l_{\varepsilon}\right)_{\varepsilon}$ belongs to $\mathcal{H}(\mathbb{R}), p_{K, \rho}\left(v_{\varepsilon}\right)$ is an $|A|$. Similarly, setting $j_{\varepsilon}(t)=i_{\varepsilon}\left(t, l_{\varepsilon}(t)\right)$ leads to $p_{K, \rho}\left(j_{\varepsilon}\right) \in\left|I_{A}\right|$. Then $\left(u_{\varepsilon}\left(\cdot, l_{\varepsilon}(\cdot)\right)\right)_{\varepsilon}$ belongs to $\mathcal{H}(\mathbb{R})$ and $\left(i_{\varepsilon}\left(\cdot, l_{\varepsilon}(\cdot)\right)\right)_{\varepsilon}$ belongs to $\left.\mathcal{J}(\mathbb{R})\right)$.

\section{Application to a characteristic Cauchy problem}

We deal with the characteristic Cauchy problem for the transport equation formally written in characteristic coordinates

$$
\frac{\partial u}{\partial t}=F(., ., u) ;\left.\quad u\right|_{\{x=0\}}=f
$$

where $f \in \mathrm{C}^{\infty}(\mathbb{R})$. We are going to formulate some assumptions which will allow us to associate to $\left(P_{c}\right)$ a generalized and well posed problem $\left(P_{g}\right)$ given below.

\subsection{From the ill-posed problem $\left(P_{c}\right)$ to a well-posed formulation $\left(P_{g}\right)$}

We approximate the characteristic curve $\{x=0\}$ by a family of non-characteristic ones $\gamma_{\varepsilon}=$ $\left\{x=l_{\varepsilon}(t)\right\}_{\varepsilon \in(0,1]}$. We assume that the family $\left(l_{\varepsilon}\right)_{\varepsilon} \in \mathrm{C}^{\infty}(\mathbb{R})^{10,1]}$ tends simply to 0 (or uniformly on each compact which is equivalent here according to Din's Theorem) when $\varepsilon$ tends to 0 and that: $\forall x \in \mathbb{R}, l_{\varepsilon}^{\prime}(x)>0$ and $l_{\varepsilon}(\mathbb{R})=\mathbb{R}$. Moreover we assume that $\left(l_{\varepsilon}\right)_{\varepsilon}$ is $c$-bounded.

Let $K \Subset \mathbb{R}^{2}$ and $a, b \in \mathbb{R}$ such that $K \subset[-a, a] \times[-b, b]$. We define

$$
\left\{\begin{array}{l}
\beta_{K, \varepsilon}=\max \left(a, l_{\varepsilon}^{-1}(b)\right) \text { and } \alpha_{K, \varepsilon}=\min \left(-a, l_{\varepsilon}^{-1}(-b)\right) ; a_{K, \varepsilon}=2 \max \left(\beta_{K, \varepsilon},\left|\alpha_{K, \varepsilon}\right|\right), \\
K_{\varepsilon}=K_{1 \varepsilon} \times K_{2} \text { with } K_{1 \varepsilon}=\left[-a_{K, \varepsilon} / 2, a_{K, \varepsilon} / 2\right] \text { and } K_{2}=[-b, b]=[-c / 2, c / 2] .
\end{array}\right.
$$

Then we have $K \subset K_{\varepsilon}$. Assume that

$$
\forall \varepsilon \in(0,1], \forall K \Subset \mathbb{R}^{2}, \forall \eta \in \mathbb{N}, \exists D_{K, \varepsilon, \eta} \in \mathbb{R}_{+}^{*}, \sup _{t \in K_{1} \varepsilon}\left|D^{\eta} f(t)\right| \leq D_{K, \varepsilon, \eta} .
$$

In addition to the previous assumptions, we collect in one formulation the sufficient conditions which allows to generate a convenient $(\mathcal{C}, \mathcal{E}, \mathcal{P})$-algebra adapted to our problem

$$
\left\{\begin{aligned}
&(i) \forall \varepsilon \in(0,1], \forall K \Subset \mathbb{R}^{2}, \forall n \in \mathbb{N}, \exists \mu_{K, n}>0, \exists M_{\varepsilon}>0, \\
& \sup _{(t, x, z) \in K_{\varepsilon} \times \mathbb{R}}\left|D^{\alpha} F(t, x, z)\right|=M_{K, \varepsilon, n} \leq \mu_{K, n} M_{\varepsilon} . \\
& \text { (ii) } \forall \varepsilon \in(0,1], \forall K \Subset \mathbb{R}^{2}, \exists \nu_{K}>0, \exists a_{\varepsilon}>0, a_{K, \varepsilon} \leq \nu_{K} a_{\varepsilon} . \\
& \text { (iii) } \forall \varepsilon \in(0,1], \forall K \Subset \mathbb{R}^{2}, \exists \xi_{K, n} \geq 0, \exists P_{\varepsilon}>0, \\
& \\
& \sup _{x \in K_{2}, k \leq n}\left|\left(l_{\varepsilon}^{-1}\right)^{(k)}(x)\right|=p_{K_{2}, n}\left(l_{\varepsilon}^{-1}\right) \leq \xi_{K, n} P_{\varepsilon}^{n} \\
& \text { (iv) } \forall \varepsilon \in(0,1], \forall K \Subset \mathbb{R}^{2}, \forall \eta \in \mathbb{N}, \exists \omega_{K, \beta}>0, \exists Q_{\varepsilon}>0, D_{K, \varepsilon, \eta} \leq \omega_{K, \eta} Q_{\varepsilon} .
\end{aligned}\right.
$$

We finally choose $\mathcal{C}=A / I_{A}$ overgenerated by: $\left(a_{\varepsilon}\right)_{\varepsilon},\left(M_{\varepsilon}\right)_{\varepsilon},\left(Q_{\varepsilon}\right)_{\varepsilon},\left(P_{\varepsilon}\right)_{\varepsilon},\left(\exp M_{\varepsilon} a_{\varepsilon}\right)_{\varepsilon}$ and $\mathcal{A}\left(\mathbb{R}^{2}\right)=$ $\mathcal{H}\left(\mathbb{R}^{2}\right) / \mathcal{J}\left(\mathbb{R}^{2}\right)$ is built on $\mathcal{C}$ with $\mathcal{E}=\mathrm{C}^{\infty}\left(\mathbb{R}^{2}\right)$ and $\mathcal{P}=\left(P_{K, l}\right)_{K \in \mathbb{R}^{2}, l \in \mathbb{N}}$.

The problem of consistency of hypotheses $(H)$ can be linked to the corresponding ones with $l_{\varepsilon}(t)=\varepsilon t$.

Example 3.1. Take $l_{\varepsilon}(t)=\varepsilon t$, then $l_{\varepsilon}^{-1}(x)=x / \varepsilon$. It is easy to see that $a_{K, \varepsilon}=2 b / \varepsilon$ and $K_{1 \varepsilon}=$ $[-b / \varepsilon, b / \varepsilon], K_{2}=[-b, b]$. For any $K \Subset \mathbb{R}^{2}$, we have

$$
\forall \varepsilon \in(0,1], \forall n \in \mathbb{N}, \exists \mu_{K, n}>0, \exists M_{\varepsilon}>0, \sup _{(t, x, z) \in K_{\varepsilon} \times \mathbb{R},|\alpha| \leq n}\left|D^{\alpha} F(t, x, z)\right|=M_{K_{\varepsilon}} \leq \mu_{K, n} M_{\varepsilon},
$$

Then $\mathcal{C}=A / I_{A}$ is overgenerated by $(\varepsilon)_{\varepsilon},\left(e^{M_{\varepsilon} / \varepsilon}\right)_{\varepsilon} ;\left(M_{\varepsilon}\right)_{\varepsilon}$.

Take now $F(x, y, z)=z /\left(1+z^{2}\right)=h(z)$. We have $\left|h^{(l)}(z)\right| \leq l$ ! for all $l \in \mathbb{N}[7]$. It follows that

$$
\forall K \Subset \mathbb{R}^{2}, \forall l \in \mathbb{N}, \max _{\alpha \in \mathbb{N}^{3},|\alpha| \leq l}\left(\sup _{(x, y, z) \in K \times \mathbb{R}}\left|D^{\alpha} F(x, y, z)\right|\right) \leq l !
$$


Consequently, we can take $m\left(K_{\varepsilon}, l\right)=l$ ! and $M_{\varepsilon}=1$. Finally $\mathcal{C}=A / I_{A}$ is overgenerated by the families $(\varepsilon)_{\varepsilon}$ and $\left(e^{\frac{1}{\varepsilon}}\right)_{\varepsilon}$. In this case, the $(\mathcal{C}, \mathcal{E}, \mathcal{P})$-algebra is actually of Colombeau type, as it is equal to the asymptotic algebra with $\left(e^{-\frac{1}{\varepsilon}}\right)_{\varepsilon}$ as asymptotic scale [6].

Theorem 3.2. Under the previous assumptions $(H), \mathcal{A}\left(\mathbb{R}^{2}\right)$ is stable under $F$ and the generalized restriction operator

$$
\mathcal{R}: \mathcal{A}\left(\mathbb{R}^{2}\right) \rightarrow \mathcal{A}(\mathbb{R}), \quad u=\left[u_{\varepsilon}\right] \rightarrow\left[u_{\varepsilon}\left(t, l_{\varepsilon}(t)\right)\right]
$$

is well defined.

Now, we can associate to $\left(P_{c}\right)$ the generalized problem $\left(P_{g}\right)$ :

$$
\frac{\partial u}{\partial t}=\mathcal{F}(u) ; \quad \mathcal{R}(u)=f
$$

\subsection{Existence of a solution to $\left(P_{g}\right)$}

In order to solve $\left(P_{g}\right)$, we begin to solve in $\mathrm{C}^{\infty}\left(\mathbb{R}^{2}\right)$ the regularized problem

$$
\left(P_{\infty}\right) \quad \frac{\partial u_{\varepsilon}}{\partial t}(t, x)=F\left(t, x, u_{\varepsilon}(t, x)\right) ; \quad u_{\varepsilon}\left(t, l_{\varepsilon}(t)\right)=f(t) .
$$

Proposition 3.3. With the assumptions $(H, 3.2)$, the problem $\left(P_{\infty}\right)$ admits a unique smooth solution $u_{\varepsilon}$ such that

$$
u_{\varepsilon}(t, x)=f\left(l_{\varepsilon}^{-1}(x)\right)+\int_{l_{\varepsilon}^{-1}(x)}^{t} F\left(\tau, x, u_{\varepsilon}(\tau, x)\right) \mathrm{d} \tau .
$$

Moreover we have the estimate

$$
\left\|u_{\varepsilon}\right\|_{\infty, K} \leq\left(\omega_{K, \beta} Q_{\varepsilon}+B_{K} a_{\varepsilon} M_{\varepsilon}\right)\left(\exp a_{\varepsilon} M_{\varepsilon}\right)^{C_{K}}
$$

where the constant $B_{K}=\mu_{K, 0} \nu_{K}, C_{K}=\mu_{K, 1} \nu_{K}$ depend only upon the compact set $K$.

This proposition comes from the Cauchy-Lipschitz theorem, applied for fixed $x$, for the existence and the uniqueness of a smooth solution $u_{\varepsilon}$ to the problem $\left(P_{\infty}\right)$, which satisfies (3.3). Starting from this relation, the Gronwall lemma, leads to the estimate (3.4).

Theorem 3.4. Under Assumption $(H)$, the problem $\left(P_{g}\right)$ admits $\left[u_{\varepsilon}\right]_{\mathcal{A}\left(\mathbb{R}^{2}\right)}$ as solution where $u_{\varepsilon}$ is the solution given in Proposition 3.3.

The proof follows the same steps as the existence results which can be found in $[7,8]$ : starting from the estimate (3.4), the proof is based on an induction process on the order of the successive derivatives that $\left(u_{\varepsilon}\right)_{\varepsilon}$ belongs to $\mathcal{H}\left(\mathbb{R}^{2}\right)$.

For linear (or semi linear) problems with irregular data, a more complete theory exists, based on the functorial properties of the Colombeau type algebras [6]. Existence and uniqueness are obtained whenever the map associating the solution to the data for the classical problem is continuously temperate. Of course, this theory fails when the problem under consideration is characteristic as in the present paper. Moreover, without further assumption the solution given by Theorem 3.4 fails in general to be unique as shown by a counter example given in [5].

\subsection{Independence of the generalized solution from the regularizing process}

The solution of all the problems which are regularized by the Colombeau method depends a priori on the choice of the regularizing process.

Any solution $u$ to $\left(P_{g}\right)$ (unique or not) depends a priori on the choice of the regularizing process thus, here, on the choice of the representative $\left(l_{\varepsilon}\right)_{\varepsilon}$ defining the map $\mathcal{R}$. We expect to obtain more precise 
informations about this dependence. A first step in this direction is done by [1] in which the purely characteristic case is studied (with regular data). By asking that $\left(l_{\varepsilon}\right)_{\varepsilon}$ belongs to $\mathcal{M}_{\tau}(\mathbb{R})$, the authors are able to prove that the generalized solution depends solely on the class of $\left(l_{\varepsilon}\right)_{\varepsilon}$ as a generalized function, not on a particular representative.

Here we have an analogous result:

Theorem 3.5. In addition to the previous assumptions, suppose that $\left(l_{\varepsilon}\right)_{\varepsilon} \in \mathcal{M}_{\tau}(R)$ and $\left(l_{\varepsilon}^{-1}\right)_{\varepsilon} \in$ $\mathcal{M}_{\tau}(\mathbb{R})$. Then, the generalized function $u=\left[u_{\varepsilon}\right]$, where $\left(u_{\varepsilon}\right)_{\varepsilon}$ is given by (3.3), depends solely on $l=$ $\left[l_{\varepsilon}\right] \in \mathcal{G}_{\tau}(\mathbb{R})$ as generalized functions and not on the representatives $\left(l_{\varepsilon}\right)_{\varepsilon}$.

For the detailed proof of the theorem 3.5, we refer to [1]. However, we shall give the main steps of the proof, as it emphasizes the difference between the case of usual Colombeau algebra and tempered generalized functions.

Lemma 3.6. Let $\left(f_{\varepsilon}\right)_{\varepsilon} \in \mathcal{M}_{\tau}(\mathbb{R})$ such that for every $\varepsilon, f_{\varepsilon}$ is bijective and $\left(f_{\varepsilon}^{-1}\right)_{\varepsilon} \in \mathcal{M}_{\tau}(\mathbb{R})$. Then, for any $\left(g_{\varepsilon}\right)_{\varepsilon} \in \mathcal{M}_{\tau}(\mathbb{R})$ such that for every $\varepsilon, g_{\varepsilon}$ is bijective, $\left(g_{\varepsilon}^{-1}\right)_{\varepsilon} \in \mathcal{M}_{\tau}(\mathbb{R})$ and $\left(g_{\varepsilon}-f_{\varepsilon}\right)_{\varepsilon} \in \mathcal{N}_{\tau}(\mathbb{R})$, we have that

$$
\left(f_{\varepsilon}^{-1}-g_{\varepsilon}^{-1}\right)_{\varepsilon} \in \mathcal{N}_{\tau}(\mathbb{R}) .
$$

Proof. We shall use the point values characterization $[11, \S 1.2 .4]$. Let $\mathcal{M}_{\mathbb{R}}\left(\operatorname{resp} . \mathcal{N}_{\mathbb{R}}\right)$ be the set of all $\left(x_{\varepsilon}\right)_{\varepsilon} \in \mathbb{R}^{(0,1]}$ such that: $(\exists N \in \mathbb{N}) \quad\left(\left|x_{\varepsilon}\right|=\mathrm{O}\left(\varepsilon^{-N}\right)\right)$ (resp. $\left.(\forall m \in \mathbb{N}) \quad\left(\left|x_{\varepsilon}\right|=\mathrm{O}\left(\varepsilon^{m}\right)\right)\right)$ as $\varepsilon \rightarrow 0$. We denote by $\widetilde{\mathbb{R}}=\mathcal{M}_{\mathbb{R}} / \mathcal{N}_{\mathbb{R}}$ the ring of generalized real numbers in the Colombeau setting. Let $\left(f_{\varepsilon}\right)_{\varepsilon},\left(g_{\varepsilon}\right)_{\varepsilon} \in$ $\mathcal{M}_{\tau}(\mathbb{R})$. Define the maps

$$
G: \widetilde{\mathbb{R}} \rightarrow \widetilde{\mathbb{R}}, \quad \tilde{x} \mapsto g(\tilde{x})=\left[\left(g_{\varepsilon}\left(x_{\varepsilon}\right)\right)_{\varepsilon}\right]_{\widetilde{\mathbb{R}}} ; \quad H: \widetilde{\mathbb{R}} \rightarrow \widetilde{\mathbb{R}}, \quad \tilde{x} \mapsto h(\tilde{x})=\left[g_{\varepsilon}^{-1}\left(x_{\varepsilon}\right)\right]_{\widetilde{\mathbb{R}}}
$$

where $g(\tilde{x})($ resp. $h(\tilde{x}))$ is the generalized point value of $g\left(\right.$ resp. $h$ ) at the generalized point $\tilde{x}=\left[\left(x_{\varepsilon}\right)_{\varepsilon}\right]$ and well defined from [11, Prop. 1.2.45]. It is easy to see that $G \circ H=H \circ G=i d$ so that $G^{-1}=H$. In the same way, if we set

$$
F: \widetilde{\mathbb{R}} \rightarrow \widetilde{\mathbb{R}}, \quad \tilde{x} \mapsto f(\tilde{x})=\left[f_{\varepsilon}\left(x_{\varepsilon}\right)\right]_{\widetilde{\mathbb{R}}} .
$$

Then $F^{-1}: \widetilde{\mathbb{R}} \rightarrow \widetilde{\mathbb{R}}$ is defined by $F^{-1}(\tilde{x})=\left[f_{\varepsilon}^{-1}\left(x_{\varepsilon}\right)\right]$.

Proving (3.5) is equivalent to prove that $f^{-1}-g^{-1}=0$ in $\mathcal{G}_{\tau}(\mathbb{R})$, and, by point value characterization [11, Prop. 1.2.47], it suffices to show that $\forall \widetilde{y} \in \widetilde{\mathbb{R}},\left(F^{-1}-G^{-1}\right)(\widetilde{y})=0$. Let $\widetilde{y}=\left[y_{\varepsilon}\right] \in \widetilde{\mathbb{R}}$. As $G$ is bijective there exists $\tilde{x}=\left[x_{\varepsilon}\right] \in \widetilde{\mathbb{R}}$ such that $\widetilde{y}=G(\tilde{x})$ and for all $\varepsilon$ we have

$$
\left(F^{-1}-G^{-1}\right)(\tilde{y})=\left[\left(f_{\varepsilon}^{-1}\left(g_{\varepsilon}\left(x_{\varepsilon}\right)\right)-g_{\varepsilon}^{-1}\left(g_{\varepsilon}\left(x_{\varepsilon}\right)\right)\right)_{\varepsilon}\right]=\left[\left(f_{\varepsilon}^{-1}\left(g_{\varepsilon}\left(x_{\varepsilon}\right)\right)-x_{\varepsilon}\right)_{\varepsilon}\right]
$$

but as $\left(g_{\varepsilon}-f_{\varepsilon}\right)_{\varepsilon} \in \mathcal{N}_{\tau}(\mathbb{R})$ we have $\left(f_{\varepsilon}^{-1} \circ g_{\varepsilon}-i d\right)_{\varepsilon} \in \mathcal{N}_{\tau}(\mathbb{R})$ so that $\left[\left(f_{\varepsilon}^{-1}\left(g_{\varepsilon}\left(x_{\varepsilon}\right)\right)-x_{\varepsilon}\right)_{\varepsilon}\right] \in \mathcal{N}_{\mathbb{R}}$, which concludes the proof.

Example 3.7. We consider the problem

$$
\left(P_{\text {char }}\right) \quad \frac{\partial u}{\partial t}=0 ;\left.\quad u\right|_{\{x=0\}}=f
$$

where $f \in \mathrm{C}^{\infty}(\mathbb{R})$. We regularize $\left(P_{\text {char }}\right)$ by choosing $l_{\varepsilon}(t)=\varepsilon t$ and obtain

$$
\left(P_{\infty}\right) \quad \frac{\partial u_{\varepsilon}}{\partial t}(t, x)=0 ; \quad u_{\varepsilon}(t, \varepsilon t)=f(t) .
$$

Clearly the solution to $\left(P_{\infty}\right)$ is the function $u_{\varepsilon}$ defined by $u_{\varepsilon}(t, x)=f(x / \varepsilon)$. Then, a generalized solution $u$ of $\left(P_{g}\right)$ is $[(t, x) \mapsto f(x / \varepsilon)]_{\mathcal{A}\left(\mathbb{R}^{2}\right)}$. Remark that here $\mathcal{C}$ is overgenerated by the family $(\varepsilon)_{\varepsilon}$ showing that $\mathcal{A}\left(\mathbb{R}^{2}\right)$ is the simplified Colombeau algebra $\mathcal{G}\left(\mathbb{R}^{2}\right)$. 
This generalized function is neither a function nor a distribution. However, it is possible to link $u$ to a distribution by means of the association process defined in Remark 2.3. Suppose that $f$ is integrable with $\int f(x) \mathrm{d} x=1$ and write

$$
\frac{1}{\varepsilon} u_{\varepsilon}:(t, x) \mapsto 1_{t} \otimes \frac{1}{\varepsilon} f\left(\frac{x}{\varepsilon}\right) .
$$

We have clearly $\lim _{\varepsilon \rightarrow 0, D^{\prime}\left(\mathbb{R}^{2}\right)}\left(u_{\varepsilon} / \varepsilon\right)=1_{t} \otimes \delta_{x}=\delta_{\Gamma}$, where $\delta_{\Gamma}$ is the Dirac distribution on the characteristic manifold $\Gamma=\left\{(t, x) \in \mathbb{R}^{2}: t=0\right\}$. Thus, the solution $u$ of the generalized problem $\left(P_{g}\right)$ associated to $\left(P_{\text {char }}\right)$ satisfies $u \underset{\varepsilon}{\sim} \delta_{\Gamma}$. In addition, this solution is not unique but depends only on the class in $\mathcal{G}_{\tau}\left(\mathbb{R}^{2}\right)$ of $(t \mapsto \varepsilon t)_{\varepsilon}$.

The change of variables $x=X-T, t=T$ turns $\left(P_{c}\right)$ into the characteristic Cauchy problem for the unidirectional wave equation

$$
\left(P_{c}\right) \frac{\partial U}{\partial T}+\frac{\partial U}{\partial X}=0 ;\left.\quad U\right|_{\{X=T\}}=v .
$$

The solution $U$ of the corresponding associated generalized problem verifies $U \widetilde{\varepsilon} \delta_{\{X=T\}}$. In other words, $U$ has a bidimensional "soliton" structure, and $\operatorname{supp} U=\operatorname{supp} \delta_{\{X=T\}}=\{X=T\}$.

\section{The framework $\mathcal{G}_{\mathcal{O}_{M}}\left(\mathbb{R}^{2}\right)$ and uniqueness}

The natural topology of $\mathcal{O}_{M}$ permits to define a new algebra of tempered generalized function, $\mathcal{G}_{\mathcal{O}_{M}}\left(\mathbb{R}^{d}\right)$ [4] which differs from $\mathcal{G}_{\tau}\left(\mathbb{R}^{d}\right)$ but permits a point value characterization [20] and an extension $\mathcal{A}_{\mathcal{O}_{M}}\left(\mathbb{R}^{d}\right)$ in the framework of $(\mathcal{C}, \mathcal{E}, \mathcal{P})$-algebras $[12]$. As $\mathcal{G}_{\mathcal{O}_{M}}\left(\mathbb{R}^{d}\right)$ is of $(\mathcal{C}, \mathcal{E}, \mathcal{P})$-type and endowed with the sharp topology [3], our goal is at least to recover uniqueness of the solution of $\left(P_{g}\right)$ in this context, the wellposedness in Hadamard setting being the final goal.

\subsection{Point values in $\mathcal{G}_{\mathcal{O}_{M}}\left(\mathbb{R}^{d}\right)$}

So first let us define $\mathcal{G}_{\mathcal{O}_{M}}\left(\mathbb{R}^{d}\right)$ as the quotient algebra $\mathcal{M}_{\mathcal{O}_{M}}\left(\mathbb{R}^{d}\right) / \mathcal{N}_{\mathcal{O}_{M}}\left(\mathbb{R}^{d}\right)$ where:

$$
\begin{aligned}
\mathcal{M}_{\mathcal{O}_{M}}\left(\mathbb{R}^{d}\right)= & \left\{\left(u_{\varepsilon}\right)_{\varepsilon} \in \mathcal{O}_{M}\left(\mathbb{R}^{d}\right)^{(0,1]}:\left(\forall \varphi \in \mathcal{S}\left(\mathbb{R}^{d}\right)\right)\left(\forall \alpha \in \mathbb{N}^{d}\right)\right. \\
& \left.(\exists M \in \mathbb{N})\left(\exists \varepsilon_{0}\right)\left(\forall \varepsilon<\varepsilon_{0}\right)\left(\sup _{x \in \mathbb{R}^{d}}\left|\varphi(x) \partial^{\alpha} u_{\varepsilon}(x)\right| \leq \varepsilon^{-M}\right)\right\} \\
\mathcal{N}_{\mathcal{O}_{M}}\left(\mathbb{R}^{d}\right)= & \left\{\left(u_{\varepsilon}\right)_{\varepsilon} \in \mathcal{O}_{M}\left(\mathbb{R}^{d}\right)^{(0,1]}:\left(\forall \varphi \in \mathcal{S}\left(\mathbb{R}^{d}\right)\right)\left(\forall \alpha \in \mathbb{N}^{d}\right)\right. \\
& \left.(\forall m \in \mathbb{N})\left(\exists \varepsilon_{0}\right)\left(\forall \varepsilon<\varepsilon_{0}\right)\left(\sup _{x \in \mathbb{R}^{d}}\left|\varphi(x) \partial^{\alpha} u_{\varepsilon}(x)\right| \leq \varepsilon^{m}\right)\right\}
\end{aligned}
$$

This definition can be compared to the one of $\mathcal{G}_{\tau}\left(\mathbb{R}^{d}\right)$. On one hand, we have $\mathcal{M}_{\mathcal{O}_{M}}\left(\mathbb{R}^{d}\right)=\mathcal{M}_{\tau}\left(\mathbb{R}^{d}\right)[4$, Prop. 3.2]. However we only have $\mathcal{N}_{\mathcal{O}_{M}}\left(\mathbb{R}^{d}\right) \supseteq \mathcal{N}_{\tau}\left(\mathbb{R}^{d}\right)$.

Example 4.1. Let $\psi \in \mathcal{D}\left(\mathbb{R}^{d}\right)$ with $\operatorname{supp} \psi \subseteq B(0,1)$ and $\psi(0)=1$. Let $e \in \mathbb{R}^{d}$ be a unit vector. Let $u_{\varepsilon}(x)=\psi\left(x-\varepsilon^{-1} e\right)$ for each $\varepsilon$. It is easy to check that $\left(u_{\varepsilon}\right)_{\varepsilon} \in \mathcal{N}_{\mathcal{O}_{M}}\left(\mathbb{R}^{d}\right)$. However $\left(u_{\varepsilon}\right)_{\varepsilon} \notin \mathcal{N}_{\tau}\left(\mathbb{R}^{d}\right)$. Indeed take $\alpha=0$. Let $p \in \mathbb{N}$ arbitrary. Then

$$
\sup _{x \in \mathbb{R}^{d}}(1+|x|)^{-p}\left|u_{\varepsilon}(x)\right| \geq\left(1+\varepsilon^{-1}\right)^{-p}\left|u_{\varepsilon}\left(\varepsilon^{-1}\right)\right| \geq\left(2 \varepsilon^{-1}\right)^{-p}|\psi(0)|=(\varepsilon / 2)^{p}
$$

so no choice of $p$ satisfies $(\forall m \in \mathbb{N})\left(\exists \varepsilon_{0}\right)\left(\forall \varepsilon \leq \varepsilon_{0}\right)\left(\sup _{x \in \mathbb{R}^{d}}(1+|x|)^{-p}\left|u_{\varepsilon}(x)\right| \leq \varepsilon^{m}\right)$.

Thus $\mathcal{G}_{\mathcal{O}_{M}}\left(\mathbb{R}^{d}\right)$ differs from $\mathcal{G}_{\tau}\left(\mathbb{R}^{d}\right)$. On the other hand, along the same lines as [4, Prop. 3.2], we get:

$$
\begin{aligned}
\mathcal{N}_{\mathcal{O}_{M}}\left(\mathbb{R}^{d}\right)=\left\{( u _ { \varepsilon } ) _ { \varepsilon } \in \left(\mathcal{O}_{M}\left(\mathbb{R}^{d}\right)^{(0,1]}\right.\right. & \mid\left(\forall \alpha \in \mathbb{N}^{d}\right)(\forall m \in \mathbb{N})(\exists p \in \mathbb{N}) \\
& \left.\left(\exists \varepsilon_{0}\right)\left(\forall \varepsilon<\varepsilon_{0}\right)\left(\sup _{x \in \mathbb{R}^{d}}(1+|x|)^{-p}\left|\partial^{\alpha} u_{\varepsilon}(x)\right| \leq \varepsilon^{m}\right)\right\} .
\end{aligned}
$$

By the same Taylor-argument as in [11, Thm. 1.2.25], we find: 


\section{Theorem 4.2.}

$$
\begin{aligned}
\mathcal{N}_{\mathcal{O}_{M}}\left(\mathbb{R}^{d}\right)=\left\{\left(u_{\varepsilon}\right)_{\varepsilon} \in \mathcal{M}_{\tau}\left(\mathbb{R}^{d}\right)\right. & \mid(\forall m \in \mathbb{N})(\exists p \in \mathbb{N}) \\
& \left.\left(\exists \varepsilon_{0}\right)\left(\forall \varepsilon<\varepsilon_{0}\right)\left(\sup _{x \in \mathbb{R}^{d}}(1+|x|)^{-p}\left|u_{\varepsilon}(x)\right| \leq \varepsilon^{m}\right)\right\} .
\end{aligned}
$$

As in the proof of Lemma 3.6, we refer to generalized points and point values as developed in [11, $\S 1.2 .4]$. We recall that $\widetilde{\mathbb{K}}=\mathcal{M}_{\mathbb{K}} / \mathcal{N}_{\mathbb{K}}$ is the ring of Colombeau generalized numbers $(\mathbb{K}=\mathbb{R}, \mathbb{C})$ and similarly $\widetilde{\mathbb{K}^{d}}=\widetilde{\mathbb{K}}^{d}$ the set of generalized points.

Definition 4.3. An element $\widetilde{x}=\left[\left(x_{\varepsilon}\right)_{\varepsilon}\right] \in \widetilde{\mathbb{R}}^{d}$ is of slow scale if

$$
(\forall n \in \mathbb{N})\left(\exists \varepsilon_{0}\right)\left(\forall \varepsilon<\varepsilon_{0}\right) \quad\left(\left|x_{\varepsilon}\right| \leq \varepsilon^{-1 / n}\right)
$$

Theorem 4.4. Let $u=\left[\left(u_{\varepsilon}\right)_{\varepsilon}\right] \in \mathcal{G}_{\mathcal{O}_{M}}\left(\mathbb{R}^{d}\right)$ and let $\tilde{x}=\left[\left(x_{\varepsilon}\right)_{\varepsilon}\right]$ be of slow scale. Then the point value $u(\tilde{x})=\left[\left(u_{\varepsilon}\left(x_{\varepsilon}\right)\right)_{\varepsilon}\right] \in \widetilde{\mathbb{C}}$ is well-defined.

Proof. Let $\left(u_{\varepsilon}\right)_{\varepsilon} \in \mathcal{M}_{\mathcal{O}_{M}}\left(\mathbb{R}^{d}\right)=\mathcal{M}_{\tau}\left(\mathbb{R}^{d}\right)$ be a representative of $u$. By [11, Prop. 1.2.45], $\left(u_{\varepsilon}\right)_{\varepsilon} \in \mathcal{M}_{\tau}\left(\mathbb{R}^{d}\right)$ implies that $\left(u_{\varepsilon}\left(x_{\varepsilon}\right)\right)_{\varepsilon} \in \mathcal{M}_{\mathbb{R}}$, and that $\left(u_{\varepsilon}\left(x_{\varepsilon}\right)-u_{\varepsilon}\left(x_{\varepsilon}^{\prime}\right)\right)_{\varepsilon} \in \mathcal{N}_{\mathbb{R}}$ if $\left(x_{\varepsilon}^{\prime}\right)_{\varepsilon}$ is another representative of $\tilde{x}$. It remains to be shown that the definition of the point value does not depend on the choice of representative of $u$. So let $\left(u_{\varepsilon}\right)_{\varepsilon} \in \mathcal{N}_{\mathcal{O}_{M}}\left(\mathbb{R}^{d}\right)$. Let $m \in \mathbb{N}$. Choose $p \in \mathbb{N}$ as in the statement of theorem 4.2. Then for sufficiently small $\varepsilon$,

$$
\left|u_{\varepsilon}\left(x_{\varepsilon}\right)\right| \leq \varepsilon^{m}\left(1+\left|x_{\varepsilon}\right|\right)^{p} \leq \varepsilon^{m}\left(2\left|x_{\varepsilon}\right|\right)^{p} \leq \varepsilon^{m}\left(2 \varepsilon^{-1 / p}\right)^{p}=2^{p} \varepsilon^{m-1} .
$$

Since $m \in \mathbb{N}$ is arbitrary, $\left(u_{\varepsilon}\left(x_{\varepsilon}\right)\right)_{\varepsilon} \in \mathcal{N}_{\mathbb{C}}$.

Theorem 4.5. Let $u \in \mathcal{G}_{\mathcal{O}_{M}}\left(\mathbb{R}^{d}\right)$. Then $u=0$ iff $u(\tilde{x})=0$ for each slow scale point $\tilde{x}$.

Proof. If $u=0$, then clearly $u(\tilde{x})=0$ for each slow scale point (since the definition of point values does not depend on the representative of $u$ ). Conversely, let $u(\tilde{x})=0$ for each slow scale point $\tilde{x}$. We first show by contradiction that

$$
(\forall m \in \mathbb{N})(\exists n \in \mathbb{N})\left(\exists \varepsilon_{0}\right)\left(\forall \varepsilon<\varepsilon_{0}\right)\left(\sup _{|x| \leq \varepsilon^{-1 / n}}\left|u_{\varepsilon}(x)\right| \leq \varepsilon^{m}\right) .
$$

Assuming the contrary, we find $M \in \mathbb{N}$, a decreasing sequence $\left(\varepsilon_{n}\right)_{n}$ tending to 0 and $x_{\varepsilon_{n}} \in \mathbb{R}^{d}$ with $\left|x_{\varepsilon_{n}}\right| \leq \varepsilon_{n}^{-1 / n}$ and $\left|u_{\varepsilon_{n}}\left(x_{\varepsilon_{n}}\right)\right|>\varepsilon_{n}^{M}$, for each $n$. Let $x_{\varepsilon}=0$ if $\varepsilon \notin\left\{\varepsilon_{n}: n \in \mathbb{N}\right\}$. Then $\tilde{x}=\left[\left(x_{\varepsilon}\right)_{\varepsilon}\right]$ is of slow scale and $\left(u_{\varepsilon}\left(x_{\varepsilon}\right)\right)_{\varepsilon} \notin \mathcal{N}_{\mathbb{R}}$, contradicting $u(\tilde{x})=0$.

Now let $m \in \mathbb{N}$ arbitrary. Choose $n$ as in equation $((4.1))$. Since $\left(u_{\varepsilon}\right)_{\varepsilon} \in \mathcal{M}_{\mathcal{O}_{M}}\left(\mathbb{R}^{d}\right)=\mathcal{M}_{\tau}\left(\mathbb{R}^{d}\right)$, there exists $N \in \mathbb{N}$ such that for small $\varepsilon$,

$$
\sup _{x \in \mathbb{R}^{d}}(1+|x|)^{-N}\left|u_{\varepsilon}(x)\right| \leq \varepsilon^{-N}
$$

Let $p=n m+n N+N$. Then, for small $\varepsilon$,

$$
\begin{array}{r}
\sup _{x \in \mathbb{R}^{d}}(1+|x|)^{-p}\left|u_{\varepsilon}(x)\right|=\max \left(\sup _{|x| \leq \varepsilon^{-1 / n}}(1+|x|)^{-p}\left|u_{\varepsilon}(x)\right|, \sup _{|x| \geq \varepsilon^{-1 / n}}(1+|x|)^{-p}\left|u_{\varepsilon}(x)\right|\right) \\
\leq \max \left(\sup _{|x| \leq \varepsilon^{-1 / n}}\left|u_{\varepsilon}(x)\right|, \sup _{x \in \mathbb{R}^{d}}(1+|x|)^{-N}\left|u_{\varepsilon}(x)\right| \sup _{|x| \geq \varepsilon^{-1 / n}}(1+|x|)^{N-p}\right) \\
\leq \max \left(\varepsilon^{m}, \varepsilon^{-N}\left(\varepsilon^{-1 / n}\right)^{N-p}\right)=\varepsilon^{m} .
\end{array}
$$

Hence $\left(u_{\varepsilon}\right)_{\varepsilon} \in \mathcal{N}_{\mathcal{O}_{M}}\left(\mathbb{R}^{d}\right)$ by theorem 4.2 . 


\subsection{The main theorem}

We start by two technical lemmas, the proof of the first one being a simple adaptation of [11, Thm 1.2.29].

Lemma 4.6. Let $\left(f_{\varepsilon}\right),\left(g_{\varepsilon}\right),\left(\tilde{f}_{\varepsilon}\right),\left(\tilde{g}_{\varepsilon}\right) \in \mathcal{M}_{\mathcal{O}_{M}}$ such that $\left[f_{\varepsilon}\right]=\left[\tilde{f}_{\varepsilon}\right]$ and $\left[g_{\varepsilon}\right]=\left[\tilde{g}_{\varepsilon}\right]$. We have that $\left[f_{\varepsilon} \circ g_{\varepsilon}\right]=$ $\left[f_{\varepsilon} \circ \tilde{g}_{\varepsilon}\right]$. If moreover $g_{\varepsilon}$ preserves slow scale points then $\left[\tilde{f}_{\varepsilon} \circ g_{\varepsilon}\right]=\left[f_{\varepsilon} \circ g_{\varepsilon}\right]$.

Lemma 4.7. Let $\left(f_{\varepsilon}\right)_{\varepsilon},\left(g_{\varepsilon}\right)_{\varepsilon} \in \mathcal{M}_{\mathcal{O}_{M}}(\mathbb{R})$ such that $f_{\varepsilon}$ and $g_{\varepsilon}$ are bijective, $\left(f_{\varepsilon}-g_{\varepsilon}\right)_{\varepsilon} \in \mathcal{N}_{\mathcal{O}_{M}}(\mathbb{R})$ and $\left(f_{\varepsilon}^{-1}\right)_{\varepsilon},\left(g_{\varepsilon}^{-1}\right)_{\varepsilon} \in \mathcal{M}_{\mathcal{O}_{M}}(\mathbb{R})$. Suppose moreover that $g_{\varepsilon}^{-1}$ preserves slow scale points. Then $\left(f_{\varepsilon}^{-1}-g_{\varepsilon}^{-1}\right)_{\varepsilon} \in$ $\mathcal{N}_{\mathcal{O}_{M}}(\mathbb{R})$.

Proof. We have $\left(f_{\varepsilon}^{-1}-g_{\varepsilon}^{-1}\right) \circ g_{\varepsilon}=f_{\varepsilon}^{-1} \circ g_{\varepsilon}-I d \in \mathcal{N}_{\mathcal{O}_{M}}$ because $g_{\varepsilon}-f_{\varepsilon} \in \mathcal{N}_{\mathcal{O}_{M}}$ which implies that $\left[f_{\varepsilon}^{-1} \circ g_{\varepsilon}\right]=\left[f_{\varepsilon}^{-1} \circ f_{\varepsilon}\right]=[I d]$. But then as $f_{\varepsilon}^{-1}-g_{\varepsilon}^{-1}=\left(\left(f_{\varepsilon}^{-1}-g_{\varepsilon}^{-1}\right) \circ g_{\varepsilon}\right) \circ g_{\varepsilon}^{-1}$ and $g_{\varepsilon}^{-1} \in \mathcal{M}_{O M}$ and preserves slow scale points, then using the preceding lemma, we find that $f_{\varepsilon}^{-1}-g_{\varepsilon}^{-1} \in \mathcal{N}_{\mathcal{O}_{M}}$.

Theorem 4.8. Suppose that $\left(l_{\varepsilon}\right)_{\varepsilon}$ is taken in the subset $\mathcal{L}_{\mathcal{O}_{M}}(\mathbb{R})$ in $\mathcal{M}_{\mathcal{O}_{M}}(\mathbb{R})$ of families $\left(g_{\varepsilon}\right)_{\varepsilon}$ such that $g_{\varepsilon}^{\prime}>0,\left(g_{\varepsilon}^{-1}\right)_{\varepsilon} \in \mathcal{M}_{\mathcal{O}_{M}}(\mathbb{R})$ preserves slow scale points, $\lim _{\varepsilon \rightarrow 0, \mathcal{D}^{\prime}(\mathbb{R})} g_{\varepsilon}=0$. Then, if $f \in \mathcal{O}_{M}(\mathbb{R})$ and $F=0$, the solution $u=\left[1_{t} \otimes f \circ l_{\varepsilon}^{-1}\right]_{\mathcal{G}_{\mathcal{O}_{M}}\left(\mathbb{R}^{2}\right)}$ of $\left(P_{g}\right)$ is unique in $\mathcal{G}_{\mathcal{O}_{M}}\left(\mathbb{R}^{2}\right)$ and depends only on $l=\left[l_{\varepsilon}\right]_{\mathcal{G}_{\mathcal{O}_{M}}(\mathbb{R})} \cdot$

Proof. Let us take $\left(l_{\varepsilon}\right)_{\varepsilon},\left(h_{\varepsilon}\right)_{\varepsilon} \in \mathcal{M}_{\mathcal{O}_{M}}(\mathbb{R})$ such that $\left[l_{\varepsilon}\right]=\left[h_{\varepsilon}\right]$ and let $u=\left[u_{\varepsilon}\right], v=\left[v_{\varepsilon}\right]$ (with $\left.\left(u_{\varepsilon}\right)_{\varepsilon},\left(v_{\varepsilon}\right)_{\varepsilon} \in \mathcal{M}_{\mathcal{O}_{M}}\left(\mathbb{R}^{2}\right)\right)$ be the corresponding solutions of $\left(P_{g}\right)$. For all $\varepsilon$, we have

$$
\left\{\begin{array}{l}
u_{\varepsilon}(t, x)=f\left(l_{\varepsilon}^{-1}(x)\right)+\mu_{\varepsilon}\left(l_{\varepsilon}^{-1}(x)\right)+\int_{l_{\varepsilon}^{-1}(x)}^{t} i_{\varepsilon}(\tau, x) d \tau \\
v_{\varepsilon}(t, x)=f\left(h_{\varepsilon}^{-1}(x)\right)+\nu_{\varepsilon}\left(h_{\varepsilon}^{-1}(x)\right)+\int_{h_{\varepsilon}^{-1}(x)}^{t} j_{\varepsilon}(\tau, x) d \tau
\end{array}\right.
$$

where $\left(i_{\varepsilon}\right)_{\varepsilon},\left(j_{\varepsilon}\right)_{\varepsilon},\left(\mu_{\varepsilon}\right)_{\varepsilon},\left(\nu_{\varepsilon}\right)_{\varepsilon} \in \mathcal{N}_{\mathcal{O}_{M}}$. First we know that $l_{\varepsilon}^{-1}-h_{\varepsilon}^{-1} \in \mathcal{N}_{\mathcal{O}_{M}}$ and $f \in \mathcal{O}_{M}$ so that $f \circ l_{\varepsilon}^{-1}-f \circ h_{\varepsilon}^{-1} \in \mathcal{N}_{\mathcal{O}_{M}}$. Furthermore, as $\mu_{\varepsilon}, \nu_{\varepsilon} \in \mathcal{N}_{\mathcal{O}_{M}}, l_{\varepsilon}^{-1}, h_{\varepsilon}^{-1} \in \mathcal{M}_{O M}$ and they preserve slow scale points, we have that $\mu_{\varepsilon} \circ l_{\varepsilon}^{-1}, \nu_{\varepsilon} \circ h_{\varepsilon}^{-1} \in \mathcal{N}_{\mathcal{O}_{M}}$. Now to finish the proof we have to check that

$$
\int_{l_{\varepsilon}^{-1}(x)}^{t} i_{\varepsilon}(\tau, x) d \tau-\int_{h_{\varepsilon}^{-1}(x)}^{t} j_{\varepsilon}(\tau, x) d \tau \in \mathcal{N}_{\mathcal{O}_{M}} .
$$

We will do it only for the first integral part, as they are almost identical. First we set, for all $\varepsilon$, $k_{\varepsilon}(t, x)=\int_{l_{\varepsilon}^{-1}(x)}^{t} i_{\varepsilon}(\tau, x) d \tau$. Let $\left(t_{\varepsilon}, x_{\varepsilon}\right)_{\varepsilon} \in \widetilde{\mathbb{R}}^{2}$ be a slow scale point. Then $x_{\varepsilon} \in \widetilde{\mathbb{R}}$ is a slow scale point and $y_{\varepsilon}=l_{\varepsilon}^{-1}\left(x_{\varepsilon}\right)$ is also a slow scale point. We have

$$
\forall \varepsilon, \exists c_{\varepsilon} \in\left[y_{\varepsilon}, t_{\varepsilon}\right], k_{\varepsilon}\left(t_{\varepsilon}, x_{\varepsilon}\right)=\int_{y_{\varepsilon}}^{t_{\varepsilon}} i_{\varepsilon}\left(\tau, x_{\varepsilon}\right) d \tau=\left(t_{\varepsilon}-y_{\varepsilon}\right) i_{\varepsilon}\left(c_{\varepsilon}, x_{\varepsilon}\right)
$$

but as $\left|c_{\varepsilon}\right| \leq \max \left(\left|y_{\varepsilon}\right|,\left|t_{\varepsilon}\right|\right),\left(c_{\varepsilon}\right)$ is also a slow scale point. But then $\left(c_{\varepsilon}, x_{\varepsilon}\right)$ is a slow scale point of $\mathbb{R}^{2}$ so that $\left(i_{\varepsilon}\left(c_{\varepsilon}, x_{\varepsilon}\right)\right)_{\varepsilon} \in \mathcal{N}_{\mathbb{R}}$ and finally $\left(k_{\varepsilon}\left(t_{\varepsilon}, x_{\varepsilon}\right)\right)_{\varepsilon} \in \mathcal{N}_{\mathbb{R}}$.

Remark 4.9. However, we cannot prove the existence of a solution to $\left(P_{g}\right)$ in $\mathcal{G}_{\mathcal{O}_{M}}\left(\mathbb{R}^{2}\right)$ if $F \neq 0$ as can be seen by taking $F(., ., u)=u$; indeed the regularized problem becomes

$$
\left(P_{\infty}\right) \quad \frac{\partial u_{\varepsilon}}{\partial t}(t, x)=u_{\varepsilon}(t, x) ; u_{\varepsilon}(t, \varepsilon t)=v(t)
$$

whose solution is $u_{\varepsilon}(t, x)=v(x / \varepsilon) e^{-x / \varepsilon} e^{t}$ which clearly is not in $\mathcal{M}_{\mathcal{O}_{M}}\left(\mathbb{R}^{2}\right)$. 


\section{The well-posedness}

Classically, in Hadamard sense, the well-posedness for a Cauchy problem asks for existence, uniqueness of solution to the problem and in addition, its continuous dependence on the data. Sharp topologies and functorial properties are extended to the case of $(\mathcal{C}, \mathcal{E}, \mathcal{P})$-algebra in [3]. Thus, one can expect here the following Hadamard setting: Let $u(v, \mathcal{R})$ be the solution given by Theorem 4.8 to the generalized problem

$$
\frac{\partial u}{\partial t}=0 ; \quad \mathcal{R}(u)=v
$$

with $v \in \mathcal{O}_{M}(\mathbb{R}) \subset \mathcal{G}_{\mathcal{O}_{M}}(\mathbb{R})$. Then, at least in a neighborhood of $v$, the map

$$
\mathcal{G}_{\mathcal{O}_{M}}(\mathbb{R}) \rightarrow \mathcal{G}_{\mathcal{O}_{M}}\left(\mathbb{R}^{2}\right), v \mapsto u(v, \mathcal{R})
$$

is continuous for the corresponding sharp topologies.

For this result, which is left to a forthcoming paper, we shall build $\mathcal{G}_{\mathcal{O}_{M}}\left(\mathbb{R}^{d}\right)$ with a unique parameter, the one used to de-characterize the problem, in contrast to previous works in which a parameter is used for the singular data, and a different one is introduced for each regularization procedure. The ring $\mathcal{C}=A / I_{A}$ will be the same for $d=1,2$.

But to obtain a good continuity result in this setting will require great care for choosing the type of tempered class of regularizations used to de-characterize the problem.

\section{References}

[1] E. Allaud, V. Dévoué. Generalized solutions to a characteristic Cauchy problem. J. Appl. Anal., 19 (2013), 1-29.

[2] J.-F. Colombeau. New Generalized Functions and Multiplication of Distributions. North-Holland, Amsterdam, Oxford, New-York, 1984.

[3] A. Delcroix. Topology and functoriality in $(\mathcal{C}, \mathcal{E}, \mathcal{P})$-algebras. Application to singular differential problems. J. Math. Anal. Appl., 359 (2008), 394-403. Doi: 10.1016/j.jmaa.2009.05.046

[4] A. Delcroix. A new approach to temperate generalized Colombeau functions. Publ. Inst. Math. Beograd, N.S., 84 (2008), no. 98, 109-121. Doi: 10.2298/PIM0898109D

[5] A. Delcroix, V. Dévoué, J.-A. Marti. Generalized solutions of singular differential problems. Relationship with classical solutions. J. Math. Anal. Appl., 353 (2009), 386-402. Doi: 10.1016/j.jmaa.2008.11.077J.

[6] A. Delcroix, D. Scarpalézos. Topology on Asymptotic Algebras of Generalized Functions and Applications. Monatsh. Math., 129 (2000), 1-14.

[7] V. Dévoué. On generalized solutions to the wave equation in canonical form. Dissertationes math., 443 (2007), 1-69.

[8] V. Dévoué. Generalized solutions to a non Lipschitz Cauchy problem. J. Appl. Anal., 15 (2009), no. 1, 1-32.

[9] Yu V Egorov. On the solubility of differential equations with simple characteristics. Russ. Math. Surv., 26 (1971), 113-130.

[10] L. Gårding, T. Kotake, J. Leray. Uniformisation et développement asymptotique de la solution du problème de Cauchy linéaire à données holomorphes; analogue avec la théorie des ondes asymptotiques et approchées. Bull. Soc. Math. France, 92 (1964), 263-361.

[11] M. Grosser, M. Kunzinger, M. Oberguggenberger, R. Steinbauer. Geometric Theory of Generalized Functions with Applications to General Relativity. Kluwer Academic Publ., Dordrecht, 2001.

[12] M. Hasler, J.-A. Marti. Functorial methods in asymptotic extensions of topological algebras. Preprint (2009).

[13] L. Hörmander. On the characteristic Cauchy problem. Ann. Math., 88 (1968), 341-370.

$[14]$ J.-A. Marti. $(\mathcal{C}, \mathcal{E}, \mathcal{P})$-Sheaf structure and applications. In: Nonlinear theory of generalized functions. M. Grosser and alii, Eds. Research notes in mathematics. Chapman \& Hall/CRC, (1999), 175-186

[15] J.-A. Marti. Non linear Algebraic analysis of delta shock wave to Burgers' equation. Pacific J. Math., 210 (2003), no. 1, 165-187.

[16] M. Nedeljkov, S. Pilipović, D. Scarpalezos. The linear theory of Colombeau generalized functions. Pitman Research Notes in Mathematics Series 385, Longman Scientific \& Technical, Harlow, 1998.

[17] M. Oberguggenberger. Multiplication of Distributions and Applications to Partial Differential Equations. Pitman Research Notes in Mathematics 259, Longman Scientific \& Technical, Harlow, 1992.

[18] M. Oberguggenberger. Generalized solutions to nonlinear wave equations. Matemàtica Contemporânea, 27 (2004), 169-187.

[19] S. Pilipovic, D. Scarpalézos. Divergent type quasilinear Dirichlet problem. Acta Appl. Math., 94 (2006), 67-82

[20] H. Vernaeve. Pointwise Characterizations in generalized function algebras. Monatsh. Math., 158 (2009), $195-213$. 